Industrial Health, 1987, 25, 183-194

\title{
Sex Differences in Mental Workload during Performance of Mental Tasks
}

\author{
Toshio KAKIZAKI \\ National Institute of Industrial Health, 21-1 Nagao 6-chome, Tama-ku, \\ Kawasaki 214, Japan
}

(Received June 4, 1987 and in revised form August 31, 1987)

\begin{abstract}
In one of two experiments which were previously carried out in male students, 24 female students performed four mental tasks (transcribing, cancelling, adding, and calculating) at maximum effort for $5 \mathrm{~min}$ each. On the basis of an objective mental worklord index, i. e., occipital midline beta-2 $\left(\mathrm{Oz} \boldsymbol{\beta}_{2}\right)$ amplitude, it was shown that all tasks caused a higher mental workload in females than males, though there were no sex differences in $O z \beta_{2}$ amplitude at rest. On a subjective mental workload index, i. e., subjective rating of task difficulty (SRTD), however, it was suggested that females had a tendency to perceive a lower mental workload in all tasks than males.

In another experiment, where a paced calculating task was imposed on another 24 female students at five grades of task load for $5 \mathrm{~min}$ each, the critical values for excessive workload were estimated to be about $7.0 \mu \mathrm{V}$ (the difference between the levels at work and at rest) for $O z \beta_{2}$ amplitude and 3.5 for SRTD. Hence, it was evaluated that none of the above four tasks constituted an objectively excessive workload in females, whereas all of them were excessive in males. Subjectively, two tasks that were seen as an excessive workload in females were not in males.
\end{abstract}

Key words : Sex difference-Mental workload-EEG (Beta-2) amplitude-Subjective difficulty rating-Task performance

\section{INTRODUCTION}

With the recent progress in technological innovations, new types of mental work have been introduced into the general workplace and have resulted in occupational stress on workers. ${ }^{1,2}$ Moreover, it is said that job overload is a major source of occupational stress. ${ }^{3)}$ In the mental health care of workers, the first problem in occupational hygiene is to keep excessive workloads in check.

Previous studies ${ }^{4-6)}$ were done to develop a physiological and psychological measure of mental workload as induced in male students during mental tasks. These studies confirmed that occipital midline beta- $2\left(\mathrm{Oz} \beta_{2}\right)$ amplitude and the subjective rating of task difficulty (SRTD) closely reflect mental activity and 
were useful indices for the evaluation of mental workload. The critical levels for excessive workload were estimated to be about $3.5 \mu \mathrm{V}$ (the difference between the values at work and at rest) for $\mathrm{Oz} \beta_{2}$ amplitude and about 4.5 for SRTD. However, it was not known whether these values would be applicable to the evaluation of mental workload in female students, because some differences between the sexes appear to exist in EEG amplitude and consciousness of workload. Accordingly, it is important to examine sex differences in mental workload by means of the above indices.

The present paper describes the sex differences in mental workload observed by comparing the experimental results obtained from female students to those previously obtained from males. $\left.{ }^{4,5}\right)$

\section{METHODS}

\section{Experiment 1 (Exp. 1)}

Twenty-four healthy female students, aged 18-24 years, served as subjects. They went to the same college as the 24 male students who were used in the previous study ${ }^{5)}$ and with whom they were compared. During the experiment, which was carried out between 2 and 5 p.m., each subject sat at a desk with her eyes open in an air-conditioned room under constant ambient conditions within the range of $23.5-24.5^{\circ} \mathrm{C}$ and $56-79 \%$ relative humidity over the course of thirteen days.

The following four mental tasks were imposed upon the subjects in the same manner as described in detail in the previous paper. ${ }^{5)}$ Transcribing (Tra): The subject was required to transcribe sentences in Japanese from a technical book to a sheet of paper using a ball-point pen. Cancelling (Can): The subject was required to check each line of Japanese syllabary characters for four specified characters on a Cancellation Test paper (published by the Institute for Science of Labor, Tokyo) and to cancel them using a pencil. Adding (Add): The subject was required to add one figure to an adjacent figure on the Uchida-Kraepelin Psychodiagnostic Test (distributed by the Psychotechnological Institute of Japan Inc., Tokyo) and to write down only the last digit of the answer in a space between the figures in question using a pencil. Calculating $(\mathrm{Cal})$ : The subject was required to perform the addition or subtraction of two two-digit numbers displayed on the Calculating Work Tester ${ }^{4}$ (Sibata Kagaku Ltd., Tokyo), and to record the last 2 digits of the answer by pressing two number keys and immediately thereafter the ENT (enter) key.

Initially, the subject practiced the above four tasks for $30 \mathrm{~min}$. Then the subject was required to perform the tasks at her own pace with maximum effort for $5 \mathrm{~min}$ for each task. Four periods of tasks and a $5 \mathrm{~min}$ rest were given to the subject in random order. This experimental session was repeated twice using different tasks. The subject was asked to perform the tasks continuously during 
the task period and was then instructed to relax with as little thinking as possible and with her eyes open during the rest period.

The measurement of EEG was made between the fourth and fifth min of each rest period and each task period in accordance with the following EEG analysis. Using the following category scale, the subjective rating of task difficulty (SRTD) that the subject perceived near the end of each task period was made after each task was executed.

\section{Experiment 2 (Exp. 2)}

Twenty-four female students (18-22 years of age), similar to those used in Exp. 1, participated in Exp. 2, at about the same time and in the same room as Exp. 1. The ambient conditions were within the range of $22.0-25.0^{\circ} \mathrm{C}$ and $54-83 \%$ relative humidity over the course of fifteen days.

After having practiced the Calculating Work Tester operation for $15 \mathrm{~min}$, each subject's work capacity for the calculating task (Cal) was determined on the basis of the number of calculations attempted in 5 min. ${ }^{4)}$ Five grades of task load of the paced calculating task-60, 70, 80, 90, and $100 \%$-were prepared for individual subjects as the percentage of work capacity. The task period for each grade was $5 \mathrm{~min}$. The subject was forbidden to repeat a calculation but was able to correct a mistake by pressing the $\mathrm{C}$ (clear) key. Five periods of the paced calculating task and a 5 min rest period were given to the subject in random order. This experimental session was repeated twice. During the rest period, the subject was allowed to relax and do as little thinking as possible while keeping her eyes open.

The number of correct responses during each 5 min task period was used to measure performance. Measurement of EEG and SRTD was made in the same manner as Exp. 1.

\section{EEG analysis}

A circular electrode was pasted on the occipital midline $(\mathrm{Oz})$ of the subject's scalp with reference to an electrode on the earlobe $\left(A_{1}\right.$ or $\left.A_{2}\right)$. A ground electrode was placed in the center of the forehead. Using the EEG-4217 (Nihon Kohden Ltd., Tokyo), a $10 \mathrm{~s}$ EEG recording was made by setting the frequency characteristics of the amplifier to a time constant of $0.3 \mathrm{~s}$ for the low range and $30 \mathrm{~Hz}$ for the high range. The EEG signals were then fed to an MCE-1100 (Nihon Kohden Ltd., Tokyo), which analyzed EEG signals in five bands ( $\delta: 2-4$, $\theta: 4-8, \alpha: 8-13, \beta_{1}: 13-20, \beta_{2}: 20-30 \mathrm{~Hz}$ ) at intervals of $0.5 \mathrm{~Hz}$ by means of fast Fourier transformation and printed out the average amplitude during each $10 \mathrm{~s}$ period for each of the five bands. Head movement and clenching of the teeth were kept to a minimum while the EEG was being recorded. Since it was confirmed in previous studies ${ }^{4-6)}$ that the $\beta_{2}$ band, among other bands, was most sensitive to the execution of mental tasks and that the response of the $\beta_{2}$ 
band was most intense at the $\mathrm{Oz}$ location, only the data on $\mathrm{Oz} \beta_{2}$ amplitudes are presented.

Scale for subjective rating of task difficulty (SRTD)

In the same manner as the previous studies, ${ }^{4-6)}$ the following category scale, similar to the ratings of perceived exertion (RPE) proposed by Borg, ${ }^{7)}$ was used to measure subjective mental workload.

1. Not difficult at all (Zenzen kitsukunai)

2. Minimally difficult (Hotondo kitsukunai)

3. Somewhat difficult (Sukoshi kitsui)

4.

5. Difficult (Kitsui)

6.

7. Considerably difficult (Kanari kitsui)

8. Very difficult (Hijōni kitsui)

9. Extremely difficult (Kiwamete kitsui)

Key words in Japanese are given in parentheses. Key words were not given to rating point 4 and 6 , for want of Japanese words appropriate to express such levels of difficulty. The subject was asked to indicate her opinion of the difficulty of performing the task using an integer from 1 to 9 .

\section{RESUlts}

\section{Experiment 1}

The mean performance levels for each task are illustrated in Fig. 1a, in comparison with those determined in male subjects. These levels are represented by the amount of task attempted in a 5 min task period because there were few errors in any work results of the four tasks. As seen in the figure, there was a significant difference between males and females in the performance levels of Tra and Can. This suggests that females might have a larger capacity for performing these two tasks successfully than males.

Figure $1 \mathrm{~b}$ illustrates the resting $\mathrm{Oz} \beta_{2}$ amplitude and the difference between $\mathrm{Oz} \beta_{2}$ amplitudes at work and at rest. No sex difference was seen in the mean resting levels (Res) of $\mathrm{Oz} \beta_{2}$ amplitude, whereas a significant sex difference was found in the mean increases of $\mathrm{Oz} \beta_{2}$ amplitude over the resting level for each of the four tasks. It did not seem that the sex difference resulted from the difference in the performance levels, as no sex difference in the performance of Add and Cal was observed. Therefore, this objective result indicates that the execution of all tasks caused more mental workload on females than on males. It is, moreover, suggested that females might invest much more mental activity in performing these tasks than males.

Figure 1c shows the mean SRTD for each task. The rating values on all tasks 
a

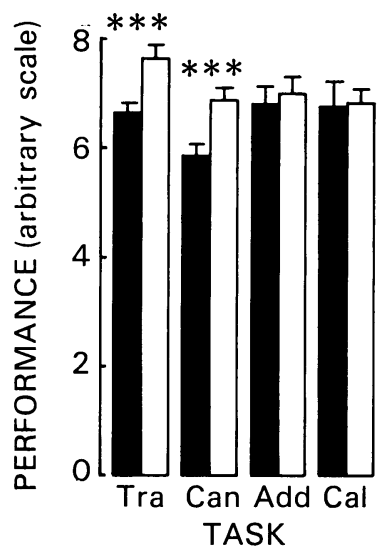

b

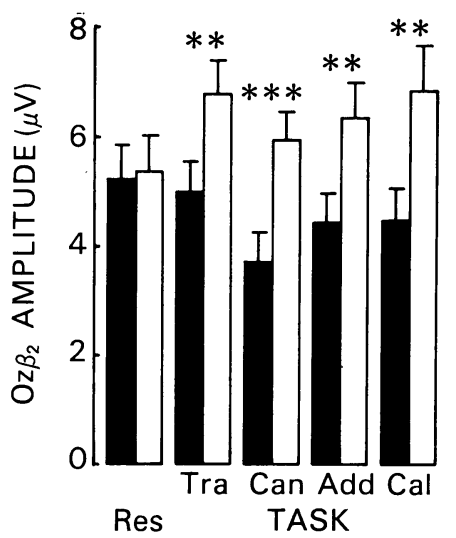

C

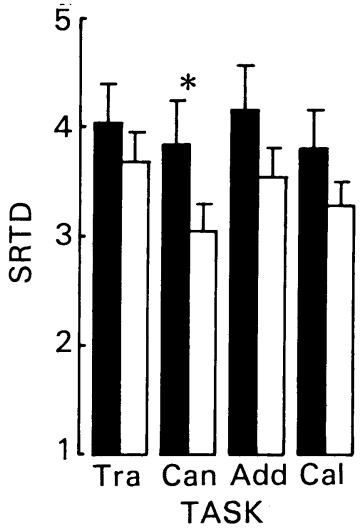

Fig. 1a, b, c. Mean values of performance, $O z \beta_{2}$ amplitude and SRTD (subjective rating of task difficulty) for males $\square$ and females $\square$ in four tasks. Res $=$ at rest. The $O z \beta_{2}$ values for the four tasks represent the difference between levels at work and at rest. Vertical lines denote SEM. ${ }^{*}: \mathbf{p}<0.05, * *: \mathbf{p}<0.01, * * *: \mathbf{p}<0.001$.

tended to be smaller for females than males, indicating that females had a tendency to perceive less mental workload in performing the tasks than males. Females may rate workload lower than males, but may not be insensitive to workload, as inferred from the following results.

Table 1 shows the results of an $F$ test of the significance of the differences in $\mathrm{Oz} \beta_{2}$ values for the four tasks. In both sex groups, the $\mathrm{Oz} \beta_{2}$ value for Can was significantly smaller than those for the other tasks (with the exception of Add in females). This objectively indicates that Can induced less mental workload in subjects of both sexes than the other tasks. This coincides with the assumption

Table 1. F test for significance of the differences in $O z \beta_{2}$ amplitudes across four tasks. Each value on the upper row represents the mean increase of $\mathrm{Oz} \boldsymbol{\beta}_{2}$ amplitude $(\boldsymbol{\mu} \mathrm{V})$ over resting level $\pm \mathrm{SD}$

\begin{tabular}{lllll}
\hline & Can & Add & Cal & Tra \\
Male & $3.66 \pm 2.52$ & $4.45 \pm 2.64$ & $4.49 \pm 2.84$ & $5.00 \pm 2.85$ \\
Female & $5.95 \pm 2.57$ & $6.38 \pm 3.08$ & $6.85 \pm 4.14$ & $6.86 \pm 2.87$ \\
\hline F test & & & & \\
Male & Can & $4.80^{*}$ & $5.40^{*}$ & $14.07^{* * *}$ \\
Female & Can & $1.34 \mathrm{NS}$ & $6.01^{*}$ & $6.09 *$ \\
Male & Add & & $0.02 \mathrm{NS}$ & $2.44 \mathrm{NS}$ \\
Female & Add & & $1.67 \mathrm{NS}$ & $1.72 \mathrm{NS}$ \\
Male & Cal & & & $2.04 \mathrm{NS}$ \\
Female & Cal & & & $0.00 \mathrm{NS}$ \\
\hline
\end{tabular}

NS: not significant, ${ }^{*}: \mathrm{p}<0.05, * * *: \mathrm{p}<0.001$ 
Table 2. F test for significance of the differences in SRTD across four tasks. Each value on the upper row represents the mean $\mathrm{SRTD} \pm \mathrm{SD}$

\begin{tabular}{lllll}
\hline & Can & Cal & Add & Tra \\
Male & $3.85 \pm 1.99$ & $3.81 \pm 1.74$ & $4.17 \pm 1.99$ & $4.06 \pm 1.69$ \\
Female & $3.06 \pm 1.24$ & $3.29 \pm 1.03$ & $3.54 \pm 1.37$ & $3.69 \pm 1.37$ \\
\hline F test & & & & \\
Male & Can & $0.05 \mathrm{NS}$ & $2.90 \mathrm{NS}$ & $1.29 \mathrm{NS}$ \\
Female & Can & $1.59 \mathrm{NS}$ & $6.96^{* *}$ & $11.84^{* * *}$ \\
Male & Cal & & $3.73 \mathrm{NS}$ & $1.85 \mathrm{NS}$ \\
Female & Cal & & $1.89 \mathrm{NS}$ & $4.75^{*}$ \\
Male & Add & & & $0.32 \mathrm{NS}$ \\
Female & Add & & & $0.64 \mathrm{NS}$ \\
\hline
\end{tabular}

NS: not significant, ${ }^{*}: \mathrm{p}<0.05,{ }^{* *}: \mathrm{p}<0.01,{ }^{* * *}: \mathrm{p}<0.001$

that Can was easier for the subjects to perform than the other tasks. Thus, mental tasks can be classified with respect to workload on basis of measured $\mathrm{Oz} \beta_{2}$ amplitude.

Another F test was made for the significance of the differences in SRTD for the four tasks (Table 2). Although no difference was seen between rating values in males, a significant difference was seen between several rating values in females. From these results it was inferred that females might be subjectively more sensitive to workload than males.

Under the present experimental conditions, the above differences in $\mathrm{Oz} \beta_{2}$ amplitude and SRTD across tasks can only be ascribed to qualitative difference in the mental tasks, since the subjects were required to perform each task with maximum effort, i.e., at their full capacity. Accordingly, it is considered that the quality of mental task is involved in workload.

\section{Experiment 2}

The mean numbers of correct responses for the calculations in each task load of the paced calculating task are shown in Fig. 2. The correct responses were linearly related to the task load up to $90 \%$ task load, but were non-linear from 90 to $100 \%$. From the figure, it was assumed that the subjects' capacity for the paced calculating task amounted to about 50 problems per $5 \mathrm{~min}$.

Figure 3 illustrates the mean $\mathrm{Oz} \beta_{2}$ amplitudes and mean SRTD for each task load. As seen in the figure, both measures regressed linearly with task load. This indicates that both the objective and subjective workload increased in proportion to the task load of the calculating task assigned to them. It seems, however, that no critical values of the measures for excessive workload can be made from this figure.

The relationship between these measures and the correct responses to calculation are shown in Fig. 4. Both measures had a linear relationship with the correct 


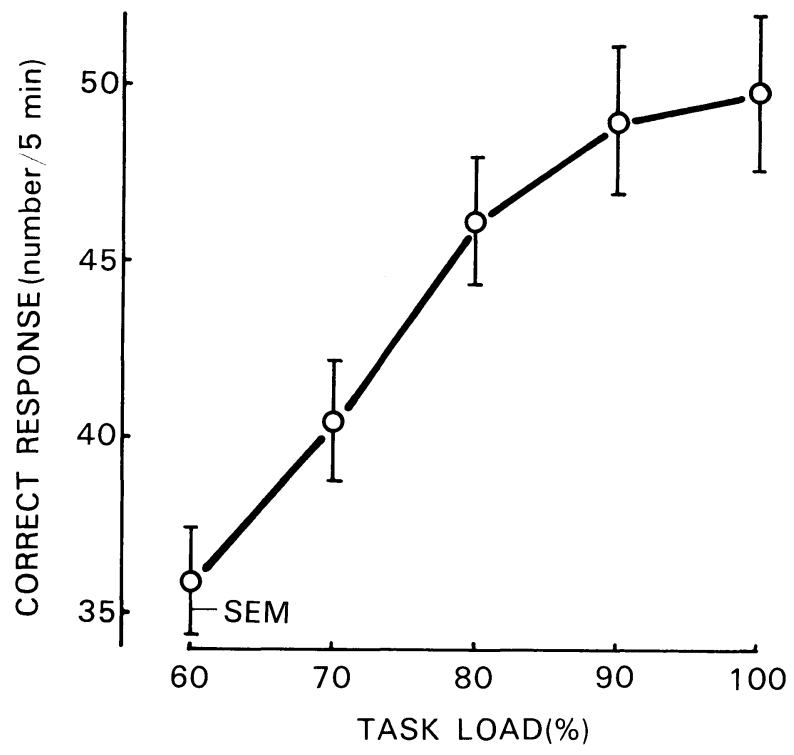

Fig. 2. Mean correct responses of calculations for each task load of the paced calculating task. The abscissa indicates the percentage of work capacity.

response up to $90 \%$ task load, but at $100 \%$ the relationship deviated remarkably from linearity. A similar relationship to this was found in a previous experiment using same procedure on male subjects. With regard to the fact that these measures represent far higher values than those proportionate to performance at above $90 \%$ task load, it was inferred that at above $90 \%$ task load the calculating task could induce excessive workload in the subjects. Hence, the critical value of $\mathrm{Oz} \beta_{2}$ amplitude for excessive workload was estimated to be about $7.0 \mu \mathrm{V}$, the difference between the amplitudes at work and at rest. Similarly, the critical value of SRTD was estimated to be about 3.5. Consequently, these values were used as the criteria for evaluating excessive workload in female subjects.

Comparison of the $\mathrm{Oz} \beta_{2}$ values given in Table 1 with the critical $\mathrm{Oz} \beta_{2}$ value indicated that none of the four mental tasks could objectively induce excessive workload in females, though all tasks caused a higher workload in females than in males. On the other hand, a comparison of SRTD (Table 2) with the critical SRTD value indicated that both Tra and Add could subjectively induce excessive workload in females, though they are apt to perceive less workload than males. Thus, a discrepancy was found between the evaluations of excessive workload based on the objective and subjective indices. There was, moreover, a sex 


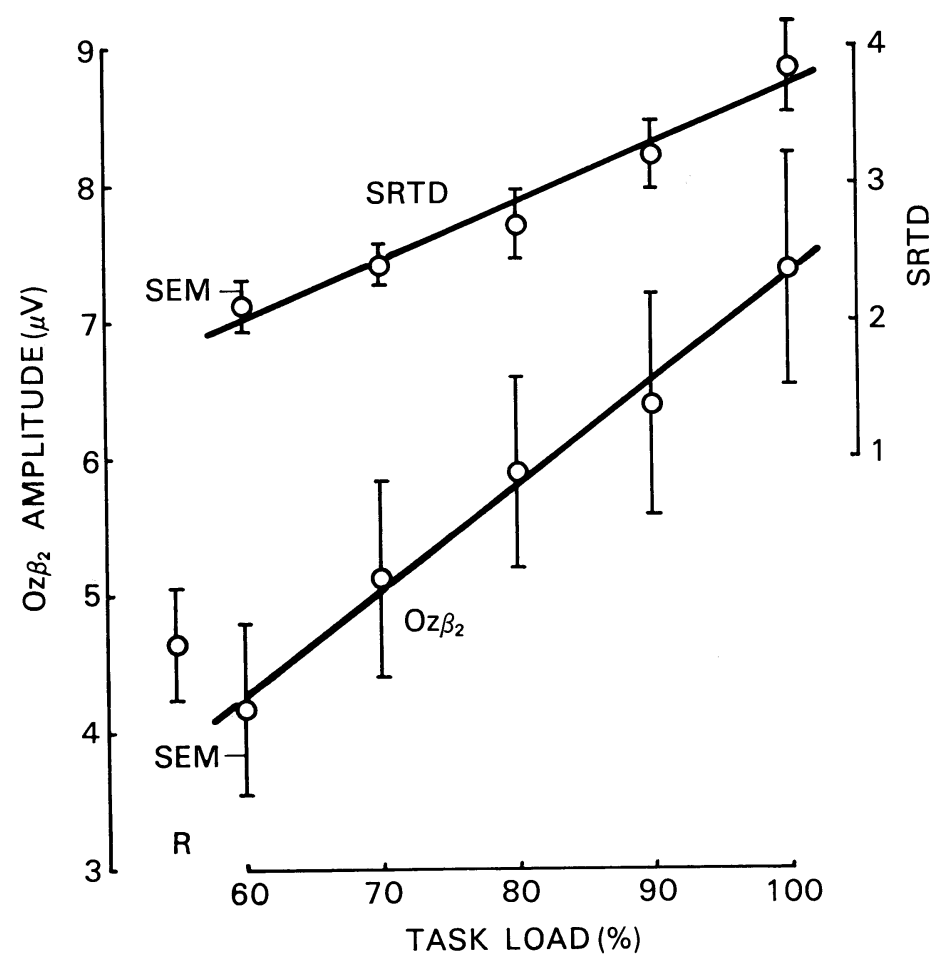

Fig. 3. Mean $O z \beta_{2}$ amplitudes and mean SRTD for each task load of the paced calculating task. The abscissa indicates the percentage of work capacity. $R=$ at rest. The $O z \beta_{2}$ values for each task load represent the difference between levels at work and at rest. Test of significance of the sample regression coefficient : $t=13.86^{* * *}$ for $\mathrm{Oz} \beta_{2}$, $\boldsymbol{t}=\mathbf{9 . 0 8}^{* * *}$ for SRTD, $\mathrm{df}=\mathbf{8}$. ${ }^{* * *}: \mathbf{p}<\mathbf{0 . 0 0 1}$.

difference in both the critical values of $\mathrm{Oz} \beta_{2}$ amplitude and SRTD for excessive mental workload.

\section{Discussion}

Almost all studies on the effect of mental tasks on scalp EEG activity have been carried out to certify EEG asymmetry associated with differences in task, sex, handedness and age. However, no consistent results have yet been found. Based on various studies, the following factors are related to sex differences in scalp EEG activity in normal human subjects at rest and at work. Concerning sex differences in EEG activity at rest, Friedl and Vogel ${ }^{8)}$ reported that women show fewer $\alpha$ waves and more $\beta$ waves than men in both $\mathrm{O}_{1}$ and $\mathrm{O}_{2}$. Matsuura et al. ${ }^{9)}$ observed less $\alpha$ continuity and percentage $\beta$ time in $\mathrm{Fp}_{1}, \mathrm{C}_{3}$ and $\mathrm{O}_{1}$, but greater percentage $\beta$ time in $\mathrm{O}_{1}$ in females than in males of an age range similar to our subjects. Flor-Henry and Koles ${ }^{10)}$ noticed larger $\beta_{2}$ amplitudes 


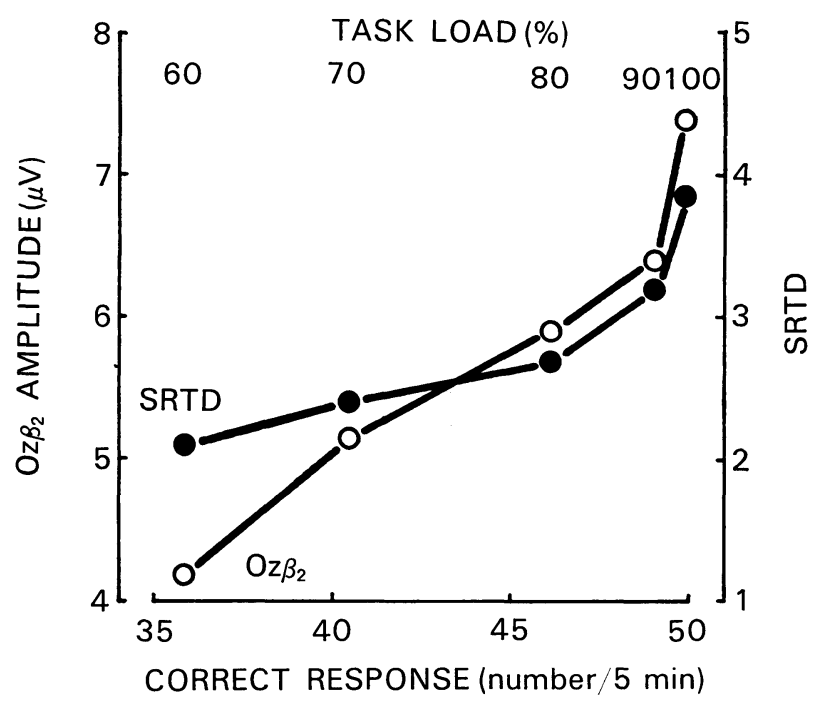

Fig. 4. Relationship between correct responses of the calculation and $\mathrm{Oz} \beta_{2}$ amplitude or SRTD. Each point corresponds to the task load put down in the figure. The $O z \beta_{2}$ values for each task load represent the difference between levels at work and at rest.

in both $T_{5}$ and $T_{6}$, and larger $\beta_{2}$ amplitude in $P_{3}$ in women than in men, but no sex difference in parietal or temporal $\alpha$ amplitude. The present study revealed no sex difference in $\mathrm{Oz} \beta_{2}$ amplitude. From these diverse results, it cannot be stated that a distinct sex difference in EEG activity at rest exists. Concerning sex differences in EEG activity at work, on the other hand, no sex difference in $\alpha$ activity was demonstrated by Flor-Henry and Koles, ${ }^{10)}$ Earle and Pikus, ${ }^{11)}$ or Glass et al. $^{12)}$ in subjects who underwent various mental tasks. Moreover, Flor-Henry and Koles ${ }^{10)}$ noticed larger $\beta_{1}$ amplitude in both $T_{5}$ and $T_{6}$, larger $\beta_{2}$ amplitude in $P_{3}$ during a spatial task, and larger $\beta_{2}$ amplitude in $P_{3}$ and $T_{5}$ during a verbal task in women than in men. However, the present study obtained significantly larger $\beta_{2}$ amplitudes in $\mathrm{Oz}$ during four mental tasks in females than males. These results indicate that sex differences in EEG activity during mental work can be seen in the $\beta_{2}$ band. In addition, it has already been shown by the previous study) in male students that the $\beta_{2}$ amplitude is most responsive to task execution in the $\mathrm{Oz}$ location. This may be accounted for by compensation for inconstant lateral asymmetry of EEG activities, since EEG activity in $\mathrm{Oz}$ represents an average of both hemispheres, precisely, over both $\mathrm{O}_{1}$ and $\mathrm{O}_{2}$. Moreover, the previous studies ${ }^{4-6}$ have confirmed that $\mathrm{Oz} \beta_{2}$ amplitude closely reflects mental activity. Thus $\mathrm{Oz} \beta_{2}$ amplitude appears to be most useful for estimating sex differences in mental workload. Using $\mathrm{Oz} \beta_{2}$ amplitude as an 
objective index of workload, the present study showed that all four mental tasks assigned caused more workload in females than males; however, they induced excessive workload in males but not in females. Hence, females may be better suited to such mental tasks than males.

A number of techniques of subjective measurement have been used to assess mental workload, as seen in the critical review by Eggemeier. ${ }^{13)}$ Above all, the Subjective Workload Assessment Technique (SWAT) ${ }^{14,15}$ ) and the Ratings of Perceived Exertion (RPE) ${ }^{7,16}$ seem to be potentially useful, in view of the fact that these were subjected to examinations of validity and utility. It may, however, be unsuitable to apply the SWAT scale to general occupational situations, since it was originally developed to assess workload in air crews and is composed of special verbal descriptions. Moreover, it is considered that all three dimensions of the SWAT scale can be represented by "task difficulty". Therefore the author adopted a category scale of "task difficulty" as a subjective measure of workload, similar to RPE, and has shown its usefulness for the subjective assessment of mental workload. Helm ${ }^{17}$ ) also observed that both the category and ratio ratings of task difficulty regressed linearly with task load, the same result as seen in Fig. 3. He indicated, moreover, that the ratio ratings more accurately reflected actual task performance than category ratings. There have been few reports on the sex difference in category ratings of workload, though many types of rating scale have been used to subjectively assess it. Using SRTD, the present study found that two tasks (transcribing and adding) were subjectively perceived as an excessive workload in females, but not in males. ${ }^{5}$ ) Since these tasks consisted of letter writing, unlike the other tasks, the subjects might have perceived them as troublesome.

Although there was a close correlation between $\mathrm{Oz} \beta_{2}$ amplitude and SRTD, a discrepancy between the estimation of excessive workload by $\mathrm{Oz} \beta_{2}$ amplitude and SRTD was found in both the present and the previous study. ${ }^{5)}$ This seems to be due to the fact that $\mathrm{Oz} \beta_{2}$ amplitude referred only to mental activity in the CNS, whereas the subjects were unable to realize the state of their own mental activity. However, it is impossible to know what measure they used when rating the difficulty of mental tasks. In physical tasks, on the other hand, Borg and Linderholm ${ }^{18}$ ) suggested that participants could sense heart rate and use it to estimate task difficulty, since there were strong correlations between heart rate and ratings of perceived difficulty.

\section{REFERENCES}

1) Fraser TM. Human stress, work and job satisfaction. ILO Occup Safety Health Ser 1983; 50: 1-67.

2) Levi L. Stress in industry. ILO Occup Safety Health Ser 1984; 51: 1-61.

3) Cooper CL. The stress of work. Aviat Space Environ Med 1985; 56: 627-32.

4) Kakizaki T. Relationship between EEG amplitude and subjective rating of task strain 
during performance of a calculating task. Eur J Appl Physiol 1984; 53: 206-12.

5) Kakizaki T. Evaluation of mental task strain based on occipital beta activity and subjective rating of task difficulty. Eur J Appl Physiol 1985; 54: 466-70.

6) Kakizaki T. Occipital midline EEG and subjective rating of task difficulty as indices of mental task strain. Eur J Appl Physiol 1987; 56: 163-8.

7) Borg G. Perceived exertion as an indicator of somatic stress. Scand J Rehabil Med 1970; 2: 92-8.

8) Friedl W, Vogel F. Geschlechtsunterschiede im normalen Ruhe-EEG bei Jungen Erwachsenen. Z EEG-EMG 1979; 10: 70-9.

9) Matsuura M, Yamamoto K, Fukuzawa H, Okubo Y, Uesugi H, Moriiwa M, Kojima T, Shimazono Y. Age development and sex differences of various EEG elements in healthy children and adults-Quantification by a computerized wave form recognition method. Electroencephalogr Clin Neurophysiol 1985; 60: 394-406.

10) Flor-Henry P, Koles ZJ. EEG characteristics of normal subjects: A comparison of men and women and of dextrals and sinistrals. Res Commun Psychol Psychiatr Behav (USA) 1982; 7: 21-38.

11) Earle JBB, Pikus AA. The effect of sex and task difficulty on EEG alpha activity in association with arithmetic. Biol Psychol (Netherlands) 1982; 15: 1-14.

12) Glass A, Butler SR, Carter JC. Hemispheric asymmetry of EEG alpha activation: Effect of gender and familial handedness. Biol Psychol (Netherlands) 1984; 19: 169-87.

13) Eggemeier FT. Current issues in subjective assessment of workload. Proc Human Factors Soc Annu Meet 1981; 513-7.

14) Reid GB, Shingledecker CA, Nygren TE. Development of a multidimensional measure of workload. Proc Internat Conf Cybern Soc 1981; 403-6.

15) Reid GB, Shingledecker CA, Eggemeier FT. Applications of conjoint measurement to workload scale development. Proc Human Factors Soc Annu Meet 1981; 522-6.

16) Borg G. Subjective aspects of physical and mental load. Ergonomics 1978; 21: 215-20.

17) Helm WR. Psychometric measures of task difficulty under varying levels of information load. Proc Human Factors Soc Annu Meet 1981; 518-21.

18) Borg G, Linderholm H. Perceived exertion and pulse rate during graded exercise in various age groups. Acta Med Scand 1967; 472: 194-206. 